\title{
De Tahrir a Wall Street por la Puerta del Sol: la difusión transnacional de los movimientos sociales en perspectiva comparada
}

\author{
From Tahrir to Puerta del Sol to Wall Street: The Transnational Diffusion of \\ Social Movements in Comparative Perspective
}

Eduardo Romanos

\section{Palabras clave}

Análisis comparativo

- Difusión de

innovaciones

- Espacio público

- Movimientos de

protesta

- Movimientos sociales

\section{Key words}

Comparative Analysis

- Diffusion of

Innovations

- Public Space

- Protest Movements

- Social Movements

\begin{abstract}
Resumen
Este artículo compara la difusión de la primavera árabe al movimiento $15 \mathrm{M}$ con la de este último a Occupy Wall Street. La comparación muestra que el contenido y el canal de la difusión fueron diferentes en uno y otro proceso. Los indignados españoles recibieron de la primavera árabe un sentido de eficacia de la acción colectiva a través fundamentalmente de medios indirectos. En el segundo proceso, la mediación de terceros facilitó la difusión de conocimientos más prácticos y complejos relacionados con nuevas formas de inclusividad y la ocupación del espacio público. La comparación sugiere que los elementos ideacionales se difunden fácilmente a través de canales indirectos mientras que las innovaciones conductuales precisan contactos personales para ser difundidas adecuadamente.
\end{abstract}

\section{Abstract}

This article examines social movement diffusion through a comparison between two processes: diffusion from the Arab Spring to the Spanish indignados, and from the latter to Occupy Wall Street. The comparison shows that the content and the channel of diffusion differ from one process to the other. The Spanish indignados received a sense of collective efficacy from the Arab Spring essentially through indirect means. In the second process, the mediation of third parties facilitated the diffusion of more practical knowledge related to new forms of inclusivity and the occupation of public space. The comparison of these two processes suggests that ideational elements are easily transmitted through indirect channels, whereas behavioural innovations require interpersonal contact to be properly transmitted.

\section{Cómo citar}

Romanos, Eduardo (2016). «De Tahrir a Wall Street por la Puerta del Sol: la difusión transnacional de los movimientos sociales en perspectiva comparada". Revista Española de Investigaciones Sociológicas, 154: 103-118. (http://dx.doi.org/10.5477/cis/reis.154.103)

La versión en inglés de este artículo puede consultarse en http://reis.cis.es

Eduardo Romanos: Universidad Complutense de Madrid | eromanos@ucm.es 


\section{INTRODUCCIÓN ${ }^{1}$}

Es un lugar común en la literatura dedicada a la reciente ola transnacional de protesta reconocer cierta conexión entre las distintas movilizaciones que la componen a nivel local. Observadores y participantes coinciden en apuntar la influencia que unas movilizaciones han podido tener sobre otras. Así, Túnez e Islandia «se han convertido en referencia para los movimientos sociales que sacudieron el orden político en el mundo árabe y desafiaron a las instituciones políticas europeas y estadounidenses» (Castells, 2012: 37). La primavera árabe influyó a su vez en el levantamiento de los indignados españoles (Flesher Fominaya, 2014; Tejerina et al., 2013; Castells, 2012) mientras que el movimiento iniciado en Madrid «impulsó al resto de Europa a participar, provocando un efecto dominó a lo largo del continente y por supuesto a través del Atlántico, con el movimiento Occupy Wall Street» (Voulgarelis, 2012: 171-172). Occupy Wall Street bebió de estas fuentes y otras más próximas, como las ocupaciones de Madison, que a su vez se vieron impulsadas, junto a un sinfín de otras nuevas ocupaciones en Estados Unidos, por las protestas organizadas en Nueva York (Kerton, 2012; Gitlin, 2012; Kroll, 2011; Hammond, 2013).

Sin embargo, una vez reconocida esta influencia se echan en falta investigaciones empíricas que indaguen en las dinámicas de difusión de unas movilizaciones a otras. Este artículo pretende contribuir a cubrir esta laguna a partir de una comparación entre dos

\footnotetext{
1 Este artículo está asociado al proyecto CSO201341035-P financiado por el Ministerio de Economía y Competitividad. Presenté una versión preliminar de este artículo en una serie de conferencias en Montreal, Barcelona, Madrid, El Escorial y París. Quiero agradecer sus interesantes comentarios a los participantes en estas reuniones, así como a Jack Hammond, Cristina Flesher Fominaya y los dos evaluadores anónimos de esta revista. También quiero agradecer a David Siddhartha Patel, Ion Bogdan Vasi y Chan S. Suh por hacerme llegar sus manuscritos y permitirme citarlos aquí, y a Sidney Tarrow por su brokerage con estos autores.
}

procesos de difusión dentro de la reciente ola de protesta: la difusión de la primavera árabe al movimiento español de los indignados (también llamado movimiento 15M), y de este al movimiento Occupy Wall Street (OWS) en Estados Unidos. El análisis abarca los nueve meses que distan entre el inicio de la primavera árabe (diciembre de 2010) y el surgimiento de OWS (septiembre de 2011), con el surgimiento del $15 \mathrm{M}$ entre medias (mayo de 2011); nueve meses de intensas movilizaciones que podemos identificar con el inicio de diversos ciclos de protesta a nivel local, en este caso en el norte de África y Oriente Medio, España y Estados Unidos.

El problema de la difusión ha recibido una gran atención en la literatura sobre los movimientos sociales (Walsh-Russo, 2014; Givan, Roberts y Soule, 2010). Cuando los investigadores del área hablan de difusión se refieren a que «algún elemento de un movimiento social (v.g., tácticas, marcos, ideologías, protestas, repertorios, campañas) se transmite entre un determinado conjunto de actores (v.g., organizaciones, redes, grupos, pueblos, comunidades, Estados) de un sistema social a través de redes directas o indirectas de comunicación» (Soule, 2013). Definiciones más estrechas sugieren que el elemento a transmitir debe ser una innovación (Rogers, 1995; Soule, 2004; Givan, Roberts y Soule, 2010). Todo proceso de difusión se compone en cualquier caso de cuatro elementos básicos: transmisor, receptor, ítem a difundir y canal a través del cual este se difunde. La comparación incluida en este artículo se centra en los dos últimos con la intención de entender mejor hasta qué punto un determinado tipo de canal es más proclive a la difusión de un determinado ítem. Sarah A. Soule (2004) planteó esta pregunta en su revisión de la literatura sobre la difusión de los movimientos sociales al mismo tiempo que animaba a los investigadores a producir comparaciones que ayudaran en su solución.

Los investigadores en movimientos sociales hace tiempo que abandonaron los 
análisis que concebían la difusión como producto del contagio, la imitación o el cálculo racional para elaborar nuevos enfoques «que entienden a quienes adoptan o rechazan las innovaciones como participantes activos (tanto individuales como colectivos) involucrados en interacciones sociales significativas» (Wood, 2012: 8). El refinamiento teórico ha traído consigo diferentes tipologías. Recientemente, Givan, Roberts y Soule (2010) se han fijado en el carácter del ítem a difundir para establecer dos tipos de difusión: conductual e ideacional. La primera se refiere a la transmisión de tácticas, formas de organización o repertorios de acción mientras que la segunda consiste en la transmisión de marcos de acción colectiva. Por su parte, en un trabajo muy influyente en el área, Tarrow (2005: cap. 6) distinguía tres tipos de difusión en función de los diferentes mecanismos subyacentes: difusión directa (o relacional), que depende de los vínculos interpersonales entre iniciadores y receptores de la innovación; indirecta (o no-relacional), que descansa en lazos impersonales fundamentalmente a través de los medios de comunicación; y mediada, que depende de la mediación de terceros que actúan como traductores o brokers entre actores que de otra forma no tendrían contacto o no reconocerían un interés mutuo entre sí. Estas tipologías y sus implicaciones serán discutidas en el análisis comparado de los dos procesos de difusión que forman los casos de estudio de este artículo.

El artículo se estructura como sigue: tras una breve presentación de las tres movilizaciones (primavera árabe, el movimiento 15M y OWS), se analizan por separado los dos procesos de difusión (de la primavera árabe al $15 \mathrm{M}$ y del $15 \mathrm{M}$ a OWS) para compararlos después en un apartado final de discusión y conclusiones que pretende avanzar en la línea de trabajo planteada por Soule. Las fuentes analizadas incluyen, además de documentos, páginas web y literatura secundaria, 30 entrevistas semiestructuradas realiza- das de forma individual a activistas del 15M y OWS en dos fases: 10 en Madrid y Barcelona entre octubre de 2011 y agosto de 2012, y 20 en Nueva York entre septiembre y octubre de 2012 (de los entrevistados, el 60\% eran hombres y el $40 \%$ mujeres). Es importante recalcar que el objeto central de esta investigación no son las movilizaciones sino los procesos de difusión entre las mismas, y en concreto los contenidos y los canales empleados en dos de esos procesos. Las entrevistas realizadas en España tenían por objetivo conocer qué se difundió allí de la primavera árabe, y a través de qué canales. En este sentido, pregunté a los activistas del $15 \mathrm{M}$ qué protestas previas les habían influido - si es que había alguna-, qué aspectos concretos de esas protestas les habían llamado la atención y cómo habían sabido acerca de ellos. Las entrevistas a los activistas norteamericanos en OWS (7 de los 20 entrevistados en Nueva York) incluyen esas mismas preguntas. El trabajo de campo en Nueva York incluye otras 13 entrevistas a activistas españoles que participaron en el surgimiento de OWS. A ellos les pregunté, entre otras cuestiones, qué aspectos del 15M les habían llamado la atención, cómo habían sabido acerca de esos aspectos y cómo los transmitieron en OWS, si es que lo hicieron. El papel desempeñado por los activistas transnacionales es precisamente una diferencia fundamental entre los dos procesos de difusión, que a su vez ayuda a entender diferencias en las innovaciones difundidas en uno y otro caso. Como se discutirá más adelante, no hubo contactos interpersonales entre los indignados y activistas o inmigrantes del norte de África y Oriente Medio lo suficientemente fuertes como para sostener la difusión directa o mediada de la primavera árabe en el 15M. En este caso, la difusión se condujo por otros canales (indirectos). También tuvo otros contenidos (más ideacionales). 


\section{MOVILIZACIONES DENTRO DE LA NUEVA OLA DE PROTESTA: PRIMAVERA ÁRABE, 15M Y OWS}

La primavera árabe es un ejemplo paradigmático de los "torrentes de lucha política» definidos por Tilly y Tarrow (2007: 211), en el que uno de los contrincantes es el gobierno, si bien en este caso lo fueron de manera simultánea los de una larga serie de regímenes. Entre los lugares clave de la movilización, seguramente el más emblemático haya sido la plaza Tahrir de El Cairo. Los manifestantes egipcios la ocuparon el 25 de enero de 2011. En la convocatoria de la protesta desempeñó un papel importante el grupo de Facebook «We are all Khaled Said», creado por Wael Ghonim (2012) en memoria de un joven activista golpeado hasta la muerte por la policía unos meses antes en Alejandría. Durante varios días, diversas marchas de protesta llegaron a la plaza desde distintos puntos de la ciudad mientras el centro de Tahrir se iba convirtiendo en una gran acampada con cierta vocación de permanencia (Patel, 2013). La plaza también fue testigo de duros enfrentamientos entre los opositores y los defensores del régimen, ayudados estos últimos por la policía. El gobierno ordenó la intervención del ejército, pero este mantuvo una posición ambigua hasta que finalmente se decantó en apoyo de los opositores, dándole la estacada definitiva al presidente Mubarak, que dimitió después de 18 días de protestas y casi treinta años en el poder.

Pocos meses después, el 15 de mayo de 2011, en la otra orilla del Mediterráneo, diversas marchas simultáneas de protesta convocadas por la plataforma Democracia Real Ya bajo el lema «No somos mercancías en manos de banqueros y políticos» consiguieron movilizar a decenas de miles de personas en toda España. En Madrid, algunos de los asistentes decidieron continuar la protesta cortando el tráfico en el centro de la ciudad con una sentada. Tras algunos enfrentamientos con la policía, que terminaron con varios de- tenidos, un grupo de unas 40 personas se quedó en la Puerta del Sol, entre otros motivos, en solidaridad con los detenidos. De esa reunión surgió una asamblea con la idea de crear y mantener una acampada permanente (Romanos, 2013). Con ella nacía acampadasol, que fue creciendo alrededor de diversas comisiones dedicadas al mantenimiento del campamento y la logística del proceso asambleario, y de diversos grupos de trabajo dedicados a generar discurso vinculado con la movilización. Los apoyos a la protesta fueron creciendo, tanto en Internet como en la Puerta del Sol y otras plazas españolas, donde se instalaron otras tantas acampadas. La Junta Electoral de Madrid prohibió las protestas durante la «jornada de reflexión» previa a las elecciones municipales y autonómicas del 22 de mayo. Alrededor de 25.000 personas desafiaron la decisión en un acto masivo de desobediencia civil. El campamento se levantó el 12 de junio, después de largas discusiones internas y la presión de las autoridades.

Cuatro días después, un grupo de neoyorquinos acampaba enfrente de su ayuntamiento en protesta contra los recortes y las políticas de austeridad del alcalde Michael Bloomberg. El conocido como Bloombergville duró tres semanas, levantándose tras la aprobación de un nuevo presupuesto municipal. Algunos de los acampados acudieron después a una reunión en 16 Beaver Street, un espacio artivista cerca de Wall Street donde inmigrantes españoles, griegos y egipcios compartieron impresiones sobre las movilizaciones que estaban teniendo lugar en sus países de origen y cómo extender la ola a ese lado del Atlántico. Algunos de los allí presentes acudieron el 2 de agosto a una protesta en Bowling Green Park, la plaza con la famosa estatua del toro de Wall Street, de donde surgió una asamblea que después pasaría a celebrarse los sábados por la tarde en una plaza del East Village (Kroll, 2011) con el nombre de New York City General Assembly (NYCGA). Las asambleas solían reunir a 50 o 
60 personas, más de la mitad inmigrantes (Lawrence, 2013). Para entonces, Adbusters ya había lanzado su convocatoria para llenar Manhattan de «tiendas, cocinas [y] barricadas pacíficas y ocupar Wall Street durante varios meses» a partir del 17 de septiembre. Ese día, miles de manifestantes marcharon por el distrito financiero de Nueva York y terminaron instalando un campamento en Zuccotti Park. Su forma recordaba a los de Tahrir y Puerta del Sol, si bien a una escala más pequeña. En su interior se celebraba una asamblea diaria facilitada en parte por quienes habían participado en NYCGA. Los occupyers crearon un slogan poderoso -We are the 99 Percent - aunque la atención mediática llegó en buena medida de la mano de la desproporcionada intervención policial, difundida a través de las redes sociales. Mientras tanto, las ocupaciones se iban diseminando por el resto del país (Vasi y Suh, 2012). El 15 de octubre, el movimiento Occupy se sumó al multitudinario Día Global de Acción lanzado por los indignados españoles. Un mes después la policía expulsaba a los acampados de Zuccotti Park.

\section{LA DIFUSIÓN DE LA PRIMAVERA ÁRABE EN EL MOVIMIENTO 15M}

\section{La difusión diferencial de los marcos de acción colectiva}

En un breve artículo, William Gamson (2011) discute la influencia de la primavera árabe en las protestas que se extendieron por Israel en el verano de 2011. Iniciadas el 14 de julio, las protestas a favor de un nuevo contrato social en Israel culminaron el 3 de septiembre con la manifestación más multitudinaria en la historia del país (Gordon, 2012). Gamson analiza las conexiones entre estas movilizaciones a partir del concepto de marcos de acción colectiva, es decir, la «acción orientada hacia conjuntos de creencias y significados que inspiran y legitiman las actividades y campañas de los movimientos so- ciales» (Snow y Benford, 1992; citado en Gamson, 2011). El concepto de marcos de acción colectiva se compone de tres elementos: el componente de injusticia, que refiere a la indignación moral expresada en la forma de conciencia política; el componente agencial, que refiere a la sensación de que es posible cambiar la situación o las políticas a través de la acción colectiva; y el componente identitario, que refiere al proceso de definición de un «nosotros» en oposición a un «ellos» con otros intereses y valores. Según Gamson (2011: 464), la influencia que la publicidad de la acción colectiva en un país puede tener en otros países se refiere casi exclusivamente al componente agencial mientras que el componente de injusticia y el identitario se basan prácticamente por entero en las condiciones locales. En la primavera árabe y las movilizaciones en Israel, el sentido de injusticia se asentaba sobre agravios diferentes y los alineamientos que la construcción de un «nosotros» inclusivo quería romper también fueron distintos. Sin embargo, el sentimiento de eficacia colectiva vinculado con las campañas de protesta no violenta y desobediencia civil viajó primero de Túnez a Egipto y luego de Egipto a Israel (Gamson, 2011).

\section{El componente agencial, a través del Mediterráneo}

¿Se dio una influencia similar entre la primavera árabe y el 15M? Veamos los tres componentes del concepto de marcos de acción colectiva por separado. Entre los agravios económicos y políticos que latían detrás de los levantamientos populares en el norte de África y Oriente Medio destacan la situación de pobreza, los altos niveles de desempleo, la brutalidad policial, la limitada libertad de expresión, la arbitrariedad del Estado y la corrupción del régimen (Kurzman, 2012). La campaña de protesta que inició el movimiento de los indignados españoles hacía referencia a algunas de estas injusticias, por ejemplo, los privilegios de la clase políti- 
ca y el desempleo, pero en buena medida sus problemas eran otros: el descontrol de las entidades bancarias, el acceso a la vivienda, el deterioro de los servicios públicos, la fiscalidad, la democracia representativa y el gasto militar (Toret, 2012: 55). La acampada instalada luego en Madrid centró sus discusiones en algunos temas con importantes diferencias con respecto a los agravios de la primavera árabe, como la reforma de la ley electoral, la separación efectiva de poderes y el control de los políticos por parte de los ciudadanos².

Con respecto al componente identitario, Gamson (2011) sugiere que en los inicios de la primavera árabe algunas organizaciones como los Hermanos Musulmanes en Egipto realizaron un esfuerzo deliberado por no enmarcar el levantamiento en términos islamistas, lo que facilitó el entendimiento con sectores desfavorecidos al margen de la intensidad o la naturaleza de sus convicciones religiosas. Goldstone (2011) también ha señalado la importancia de coaliciones interclasistas e interregionales en Túnez, Egipto y Libia. En España, como en general en las fases iniciales de los ciclos de protesta (Tarrow, 1989), los activistas optaron igualmente por la construcción de un «nosotros» inclusivo, aunque aquí el proceso fue en buena medida diferente: la identificación de unos responsables claros (las élites políticas y económicas, en coalición para defender sus intereses) y la elaboración de demandas generales facilitaron la participación de una gran parte de la población. La posible reticencia de personas sin una experiencia previa en movimientos sociales se vio reducida por una serie de elementos que animaban a la participación, por ejemplo, la celebración de asambleas abiertas, la ausencia de símbolos partidistas, la creación de comisiones de respeto y el anonimato (en la red y la plaza).

2 http://madrid.tomalaplaza.net/2011/05/26/acampadasol-consensua-cuatro-lineas-de-debate/
Mientras que los procesos de construcción de un sentido de injusticia y de una identidad colectiva en el movimiento $15 \mathrm{M}$ estuvieron anclados en las condiciones locales, el componente agencial de los marcos de acción colectiva parece que estuvo, como en el caso de Israel, fuertemente influenciado por la primavera árabe. Así lo reconoce Javier Toret (2012: 55), uno de los organizadores de la protesta del 15 de mayo:

La manifestación pretendía vincularse a los movimientos emergentes en Europa... Pero si algo impulsó la convocatoria fue la «Primavera árabe». La fuerza contagiosa de esas revueltas inspiró a muchas personas a creer que era posible rebelarse.

La influencia de Tahrir se dejó sentir también en las acampadas levantadas tras la manifestación del 15 de mayo. Preguntado sobre qué pensaba que iba a ocurrir después de la primera noche en la Puerta de Sol, uno de los acampados contesta:

Lo que había ocurrido en Egipto, que la gente había salido y había tomado las plazas... nos parecía que era algo que podía ocurrir [en España]. Evidentemente no imaginábamos que iba a ocurrir a ese nivel... pero sí veíamos que podía ser realizable... Para mí, eso tiene mucha fuerza ${ }^{3}$.

Tras la caída de Mubarak, los activistas españoles fueron conscientes de la eficacia de una acción colectiva que utilizaba de forma habilidosa las redes sociales y al mismo tiempo se hacía fuerte en las plazas. Fue lugar común entre los indignados pensar que sin Facebook, Twitter y YouTube la primavera árabe no hubiera sido posible. Más allá del papel real de las redes sociales en esa movilización (Diani, 2011), los integrantes de Democracia Real Ya vieron en los tuiteros egipcios el tremendo potencial del uso político de

\footnotetext{
3 Entrevista a Miguel (29 años, doctorando en física teórica), 6/3/2012, Madrid.
} 
la tecnología, no tanto para transmitir información sino como forma de «organización interactiva» (Muñoz, 2011: 42). Los usos no eran nuevos, pero sí parecía serlo su capacidad de movilización. Por otro lado, la ocupación de Tahrir se convirtió en un símbolo que luego los indignados reprodujeron a su manera en la Puerta del Sol. Antes que los israelitas y los estadounidenses, los españoles fueron los primeros en sacar masivamente sus tiendas de campaña a la calle y ocupar con ellas las plazas centrales de sus ciudades, reproduciendo con ello las técnicas de desobediencia civil no violenta que habían visto en Tahrir.

\section{¿Cómo viajó y fructificó la agencia?}

Los indignados españoles recibieron la información de la acción colectiva en Egipto y otros países de la zona a través de la publicidad alcanzada en los medios de comunicación, y sobre todo en las redes sociales. Los testimonios de los activistas recogidos en las entrevistas sugieren que no hubo contactos interpersonales, o que si los hubo fueron muy débiles. Esto no quiere decir que el conocimiento alcanzado por los indignados españoles respecto a la primavera árabe fuera superficial. Durante cuatro meses, los organizadores de la campaña de Democracia Real Ya estuvieron investigando a los tuiteros egipcios y la forma en la que utilizaban las nuevas tecnologías (Muñoz, 2011). Como se ha sugerido anteriormente, las redes sociales servían precisamente para fomentar un sentido de conectividad que los potenciales activistas no encontraban en los medios de comunicación convencionales. En este sentido, Carolina, hacker y participante en acampadasol, señala cómo:

El uso de la tecnología que hacían, el tener activos los Twitters, el tener activo YouTube, de alguna manera sintonizaba un poco conmigo y me decía: «Aquí me está transmitiendo algo directamente la gente. No estoy siendo mediatizada, ni siquiera por un medio alternativo, sino que de repente lo estoy viendo, no sé [exactamente] qué está pasando pero me está llegando»4.

Gamson (2011: 467) sostiene que el sentido de eficacia colectivo, una vez difundido, se ve fuertemente influenciado por la respuesta de las autoridades. Si fallan las medidas de control, el componente agencial aumenta. Es lo que ocurrió en Tahrir, donde el ejército rehusó reprimir a los manifestantes. También en la Puerta del Sol, donde la policía desalojó el campamento en la segunda noche pero se vio desbordada por la reacción ciudadana y finalmente las autoridades decidieron no seguir empleando la fuerza, lo que de alguna forma dio alas a la movilización. Sin embargo, no solo la influencia de otras movilizaciones y el fracaso de las medidas de control social aumentan el sentido de agencia colectiva. También lo hace la propia movilización, que contribuye a crear un estado de esperanza en el que, si no todo, muchas cosas son posibles (Romanos, 2011b). En el caso de los indignados españoles, el éxito temprano de su movilización en términos de participación y repercusión mediática les llevó a algunos a pensar que en «esa primera semana se podían levantar todas las plazas del mundo»5. El sentido de agencia colectiva aumentó, y los indignados pasaron de estar «anestesiados» o «paralizados» a participar en la acción colectiva (Álvarez et al., 2011). De ahí la placa colocada en la Puerta del Sol con el lema: «Dormíamos, despertamos. Plaza tomada».

\section{LA DIFUSIÓN DEL 15M EN OWS}

Tras analizar la difusión de Egipto a España, este apartado se centra en la difusión de Es-

\footnotetext{
4 Entrevista por videoconferencia a Carolina (8/4/2012).

5 Entrevista de Stéphane M. Grueso con Miguel. Disponible en http://madrid.15m.cc/2011/12/conversaciones15mcc-miguel-arana.html> (acceso: 15/2/2013).
} 
paña a Estados Unidos. Seleccionar y estudiar estos dos procesos por separado no quiere decir que estos no se hayan simultaneado, o que no se hayan dado otros procesos de difusión dentro de la reciente ola transnacional de protesta. El levantamiento popular de los egipcios tuvo una influencia innegable en el surgimiento de Occupy Wall Street. De hecho, el llamamiento de Adbusters comenzaba preguntando: "Are you ready for a Tahrir moment?». El componente agencial viajó de Egipto a Nueva York, empujado a su vez por la visibilidad que la movilización de los indignados españoles tuvo a nivel internacional. Sin embargo, la difusión de la primavera árabe a los indignados españoles parece haber sido en algunos aspectos diferente de la difusión de los indignados a Occupy Wall Street.

\section{Los inmigrantes españoles en Nueva York}

Una diferencia importante entre los dos procesos de difusión consiste en el papel desempeñado por los activistas transnacionales, y en particular los emigrantes españoles residentes en Nueva York. Meses antes del surgimiento de OWS, los inmigrantes españoles crearon una red activista en la ciudad. El 21 de mayo de 2011, varios centenares se manifestaron en Washington Square vinculando su protesta con las que ese día tenían lugar en la Puerta del Sol de Madrid y otras plazas españolas. La convocatoria partió de un grupo de Facebook con el nombre de Democracia Real Ya - Nueva York creado tras el éxito de las manifestaciones del 15 de mayo en España. El grupo creció rápidamente en la red y aproximadamente sesenta de sus miembros se vieron las caras por primera vez en una sala de la Universidad de Columbia el 19 de mayo. La elección del lugar no es casual. Entre los inmigrantes españoles movilizados había gran densidad de estudiantes e investigadores universitarios. Algunos de ellos entendieron el movimiento de las plazas españolas como parte de una ola de protesta más amplia en relación con problemas globales y se organizaron para extender la moviliza- ción a su país de acogida, buscando resonancia en medios y grupos locales. Con el paso del tiempo se convirtieron en un grupo de afinidad que participó de manera muy activa en las movilizaciones previas a la ocupación de Zuccotti Park (Kroll, 2011; Graeber, 2011; Lawrence, 2013). Antes de la ocupación, algunos de ellos organizaron una charla en una librería y centro activista en Lower East Side, para explicar lo que estaba pasando en España. Los emigrantes españoles seguían las movilizaciones a través de medios de comunicación convencionales, redes sociales y comunicaciones interpersonales con familiares y amigos que participaban en ellas. Algunos de ellos también viajaron a España y pudieron participar brevemente en las movilizaciones. Como se verá más adelante, esas experiencias les sirvieron para adquirir un conocimiento que luego transmitieron en el nuevo contexto local.

La charla en la librería fue el primer paso en un esfuerzo de conexión con activistas locales. Poco después visitaron Bloombergville, donde los inmigrantes españoles mantuvieron conversaciones con los acampados, algunos de los cuales recibieron la información sobre las protestas en España con una mezcla de «incredulidad, desconocimiento y también un punto de entusiasmo". Parece ser que en el encuentro en 16 Beaver hubo más receptividad. Allí se estableció cierta sintonía, que después se fortaleció en el desarrollo de New York City General Assembly (NYCGA), donde algunos españoles entraron en el grupo de trabajo de difusión «con la idea de que [este] tenía que ser un movimiento para gente que no fuera activista sino para todo el mundo»6.

\footnotetext{
6 Entrevista a Luis Moreno-Caballud (36 años, profesor universitario), 6/10/2012, Nueva York. Según Lawrence (2013), los españoles solían representar entre un 10-20\% de los asistentes a las reuniones de la NYCGA. La mayoría de estos españoles tenían formación académica de posgrado y carecían de experiencia activa previa en movimientos sociales.
} 


\section{Innovaciones organizativas y problemas de traducción}

Como se ha mencionado anteriormente, la inclusividad es un valor central en el 15M. Sin embargo, en modo alguno es algo nuevo en el campo de los movimientos sociales. Podemos encontrar formas de inclusividad organizativa en los movimientos post-1968, por ejemplo el de mujeres. Della Porta (2005) señala cómo los activistas del movimiento antiglobalización adoptaron este principio de una forma todavía más intensa, siendo una de las características básicas del modelo de democracia deliberativa practicado en sus redes. Dicho modelo promueve la inclusión de la extraordinaria diversidad de los actores que componen este «movimiento de movimientos» en las decisiones que se adoptan en su seno. Para ello se establecen mecanismos como la asamblea general, en la que cada uno puede expresar sus opiniones.

El movimiento de los indignados adoptó básicamente el modelo de democracia deliberativa (Romanos, 2011a). Sin embargo, podemos advertir dos aspectos de la inclusividad que resultan hasta cierto punto novedosos; aspectos que a su vez fueron transmitidos a los activistas en Estados Unidos. En primer lugar, la inclusividad promovida por los indignados no tiene tanto que ver con los ya participantes en el movimiento $-y$ el establecimiento de mecanismos que aseguren su inclusión en el proceso de toma de decisiones - sino con los potenciales participantes. Aquí la plaza juega un papel importante. Una de las novedades del 15M residiría en colocar en el centro del espacio público la experimentación con un nuevo modelo de democracia. En ese sentido, este movimiento impulsó el traslado de prácticas de democracia deliberativa desde recintos más o menos limitados (v.g., campamentos, foros sociales o centros sociales) a las plazas, invitando a que los transeúntes se unieran a este ejercicio, en lo que parece ser una diferencia importante con respecto a movimientos y movilizaciones anteriores. Como señala Lawrence (2013), el cambio de espacio supone un cambio de orientación del movimiento; el foco de atención se pone en la gente normal y corriente de fuera de la asamblea y no tanto en las actividades de los ya involucrados en esos encuentros. El mismo Lawrence (2013: 9) fue testigo de cómo los emigrantes españoles promovieron esta forma de inclusividad en la NYCGA:

Durante las asambleas en Tompskins Square Park, mientras buena parte del debate se centraba en cuestiones tácticas y logísticas en relación con la ocupación, se podía ver a menudo a [Begoña] Santa-Cecilia [una artista española afincada en Brooklyn] distribuyendo flyers entre los curiosos que pasaban por el parque, a los que les hablaba sobre el fundamento de Occupy. La idea era que la asamblea debía permanecer abierta al $99 \%$ de la población, que se consideraba como el protagonista real del movimiento, más que encerrarse en sí misma como una pequeña vanguardia revolucionaria.

El segundo aspecto tiene que ver con un significado menos racional y más afectivo de la inclusividad, no tan orientado hacia el proceso de toma de decisiones sino hacia la transformación del espacio público como un espacio abierto también a la empatía. En agosto de 2011, algunos indignados reflexionaban sobre las características básicas del movimiento en España, siendo una de ellas la «INCLUSIVIDAD. La fuerza de este movimiento es ser muchos y distintos [...] Los espacios que nos dan fuerza, que nos alegran y potencian, son los que permiten a cada cual sentirlos como propios»7. Según algunos emigrantes españoles en Nueva York, este concepto de inclusividad, que podemos llamar inclusividad basada en la empatía, o inclusividad empática, no se fomentaba en NYCGA. La asamblea se orientaba más hacia cuestiones estratégicas, lo que,

7 http://madrid.tomalaplaza.net/2011/08/12/ 
en su opinión, podía representar un problema en la apertura del movimiento hacia personas sin una participación previa en movimientos sociales:

Lo que no veíamos [en NYCGA] era lo que había en España de afectivo, de hablar con una persona a la que le pasan cosas, que está mal... Entonces yo me puse la tarea de decirlo en cada asamblea. Si hacía una intervención, era para decir: «aquí no estamos solo para hablar de cosas, o para hacer planes, somos personas a las que nos pasan cosas y aquí están en juego sentimientos». Me parecía que era muy importante. Además, se montaban las típicas trifulcas de siempre por egos. Y a mí me parecía, a nosotros nos parecía, que si no había esa cosa acogedora que tenía el 15M, esa cosa empática, [el movimiento] no nos interesaba.

Quien habla es Luis Moreno-Caballud, que al ser preguntado por la recepción del mensaje, comenta la sensación de que esas palabras «no eran recibidas con la incredulidad o incomprensión con que a veces se recibían otras cosas cuando hablábamos sobre España»8.

Sin embargo, el proceso de transmisión de este concepto de inclusividad se topó con un obstáculo habitual en los procesos de difusión derivado de la utilización de lenguajes diferentes por parte de diferentes activistas (Doerr, 2008; Chabot, 2010; Wood, 2012). Un ejemplo de este tipo de problemas fue la elaboración de un mensaje por parte del grupo de difusión de OWS dirigido a atraer participantes a la cita del 17 de septiembre lanzada por Adbusters. El mensaje identificaba un problema social no tanto en «la dominación de nuestra economía y nuestro gobierno por parte de las multinacionales» sino en los efectos de esa dominación sobre «nuestras vidas y comunidades». Durante su lectura en la asamblea, algunos activistas con expe-

8 Entrevista a Luis Moreno-Caballud, 6/10/2012, Nueva York. riencia de participación en movimientos sociales de la década de 1960 criticaron la utilización en la propuesta de conceptos de alguna forma oxidados y que remitían a ese ciclo de protesta, como «empoderamiento». Finalmente la asamblea decidió que la propuesta fuera reformulada. Un testigo del proceso explica cómo en general el discurso «emocional» de los españoles era bastante rompedor, pero venía de un español traducido que provocó precisamente las críticas. En su opinión, «lo que hacen básicamente [los activistas locales] es rechazar ese documento por su lenguaje pero incorporar mucho el mensaje»9. En este sentido, los transmisores y receptores mantuvieron una comunicación que modificó la forma del mensaje antes de que este fuera finalmente adoptado (Chabot, 2010). Los inmigrantes contribuyeron a la elaboración de un mensaje que no fue adoptado hasta que utilizó un lenguaje que tuviera sentido para los potenciales receptores. Una vez traducido en ese lenguaje, los receptores pusieron en práctica las innovaciones, completando así el proceso de difusión.

\section{Cómo ocupar, y cómo darle sentido a la ocupación}

Tras el 17 de septiembre, los españoles continuaron apuntalando la apertura de OWS a la manera del 15M. En este sentido destacan dos iniciativas. La primera fue instalar una serie de mesas que dieran la bienvenida a la gente y les informaran de la actividad en Zuccotti Park. La iniciativa partió de dos activistas que habían ido a Madrid y conocido allí el puesto de información de acampadasol:

Si no hubiéramos visto ese puesto de información, no nos hubiéramos enterado de nada... No hubiéramos sabido para qué éramos útiles, qué podía-

\footnotetext{
9 Entrevista a Jeff Lawrence (28 años, doctorando), 16/10/2012, Nueva York.
} 
mos hacer... Había mucha información y había gente allí para informarte de lo que estaba pasando en la ciudad con relación a[l 15M]. Eso era la idea que teníamos. La información es lo más importante. Welcome the people es lo más importante en esta plaza ${ }^{10}$.

Isham Christie (27 años, estudiante de master), activista norteamericano primero en NYCGA y luego en OWS, señala hasta qué punto el puesto de información organizado por los inmigrantes españoles en Zuccotti Park fue importante:

Fue justo después de que nos hiciéramos realmente populares en los medios y de que viniera tanta gente. La gente no sabía cómo conectarse. [Los españoles] fueron muy útiles ya que trajeron unas cuantas mesas [desde las que decir] «estos son los diferentes grupos de trabajo". Y eso fue una contribución inmensa. Seguramente no aparezca en los libros de historia, pero a partir de entonces la gente tenía un lugar al que ir y participar11.

La segunda iniciativa fue la organización de los open forum: «una discusión en Liberty Square en donde un ponente voluntario ofrece una breve presentación importante para la protesta seguida de una discusión abierta»12. Por allí pasaron Naomi Klein o Slavoj Žižek, entre otros. Siguiendo el ejemplo de lo que habían visto en el movimiento $15 \mathrm{M}$, los emigrantes españoles quisieron ir más allá de la ocupación proponiendo actividades que le dieran sentido: «Se hacía el énfasis en el hecho de ocupar pero nadie parecía concebir que esa plaza había que utilizarla para algo»13. A juzgar por testimo-

\footnotetext{
10 Entrevista a Begoña Santa-Cecilia, 4/10/2012, Nueva York.

11 Entrevista a Isham Christie, 11/10/2012, Nueva York.

12 http://www.nycga.net/groups/education-and-empowerment/docs/minutes---empowerment-and-education-working-group-meeting---12211---60-wall-st

13 Entrevista a Vicente Rubio (32 años, doctorando), 27/9/2012, Nueva York.
}

nios de activistas norteamericanos, parece ser que sus esfuerzos no fueron en balde:

La idea de mantenerse activo en la ocupación, de exigir trabajo de la gente, pero no de manera abusiva sino de un sentimiento colectivo de trabajo, de comisiones, de que había mucho que hacer. Que esto no era solo para quedarse a dormir, sino que esto es una ocupación muy activa. Ese sentimiento vino de los españoles ${ }^{14}$.

Con el paso del tiempo, el grupo promotor de los open forum dirigió sus esfuerzos a conectar la actividad de la plaza con la de grupos comunitarios y activistas que estuvieran trabajando en otros puntos de Nueva York. Tras el desalojo de Zuccotti Park, el grupo pasó a llamarse Making Worlds, cuyos miembros siguen siendo predominantemente españoles.

Los emigrantes españoles en Nueva York contribuyeron también a la transmisión de otras innovaciones a partir de la distribución de documentos creados por los indignados españoles. El más importante fue Cómo Cocinar una Revolución Noviolenta, que explicaba la organización interna de las acampadas (en comisiones, grupos de trabajo y asambleas generales) y el proceso horizontal de toma de decisiones. También incluía otras informaciones, por ejemplo, sobre quiénes eran y por qué se movilizaban los indignados, además de un organigrama-mapa de acampadasol. Sus autores fueron los miembros de World Extention Team, una comisión creada en los primeros días de la acampada en Madrid con el objetivo de contribuir a la coordinación de un movimiento de protesta a nivel internacional. Los españoles tradujeron y distribuyeron el documento en NYCGA y OWS. También se publicó en diversas páginas web, entre ellas takethesquare.net. Sin embargo, a juzgar por los testimonios recogidos en las entrevistas, su recepción en Es-

14 Entrevista a Justin Wedes (26 años, educador), 6/10/2012, Nueva York. 
tados Unidos se apoyó en los contactos interpersonales creados por los emigrantes españoles y otros activistas transnacionales: estadounidenses que habían participado en el movimiento en España y que luego participaron en el surgimiento y desarrollo de OWS. Según activistas y observadores locales, estos materiales fueron relevantes en Zuccotti Park, sobre todo en relación con el funcionamiento de la asamblea general y la organización del espacio en la acampada15.

\section{Difusión transnacional y contienda global}

En cualquier caso, las prácticas difundidas por los emigrantes españoles no fueron la única fuente de organización de la democracia en Zuccotti Park. En OWS participaron otros activistas con otras experiencias de horizontalismo que también sirvieron de inspiración, por ejemplo, en relación con el movimiento antiglobalización o las protestas argentinas en 2001 (Sitrin y Azzellini, 2012; Naidu, 2011). Esta confluencia de experiencias remite a un campo global de contienda a tener en cuenta a la hora de analizar procesos específicos de difusión transnacional. Además de las innovaciones específicas aprendidas a partir de una movilización en concreto, los activistas se ven influenciados por todo un imaginario global de discursos y prácticas contenciosas. En cualquier caso, la referencia a otras experiencias de horizontalismo nos ayuda a entender algunas diferencias entre los procesos deliberativos en Zuccotti Park y las plazas españolas. Por ejemplo, el «progressive stark» diseñado para asegurar que los grupos desfavorecidos tengan la oportunidad de hablar no estaba tan formalizado en España, y el consenso allí debía ser total, lo que sin duda bloqueó la aprobación de muchas propuestas, mientras que en Nueva York se practicaba un consenso modificado que aceptaba pequeñas disiden-

15 Entrevista a Isham Christie, 11/10/2012, Nueva York. Véase también Hammond (2013). cias. Otras diferencias tienen que ver con el contexto político, y en concreto con el control policial de la protesta. En este sentido, la prohibición de cualquier tipo de equipo de sonido en Zuccotti Park obligó a la utilización del «micrófono humano», con todo lo que ello significa: más participación y cercanía pero al mismo tiempo menos fluidez y sofisticación en la elaboración de argumentos.

Además de los emigrantes españoles y de los estadounidenses que viajaron a España, otros activistas transnacionales parecen haber servido de vínculo importante entre los movimientos de España y Estados Unidos. Son los españoles (y residentes en España) que acudieron con motivo de la ocupación de Zuccotti Park. Estos activistas viajaron a Nueva York a título personal y sin estar coordinados. El viaje no fue pagado por el movimiento, ya que, a diferencia de OWS, el 15M no recibía donaciones y no disponía de fondos. Los testimonios de activistas estadounidenses recogidos en las entrevistas señalan que estos «visitantes» españoles contribuyeron al desarrollo del movimiento con la transmisión de diversas cuestiones prácticas con resultados importantes en el surgimiento y continuidad del movimiento, por ejemplo, en relación con la forma de hacer frente a problemas similares, como los planteados por grupos que ponían en peligro la convivencia en la plaza ${ }^{16}$, o cuestiones como el abastecimiento de energía para el campamento o el diseño y montaje del equipo de media encargado, entre otras cosas, del funcionamiento del live-streaming ${ }^{17}$.

\section{Discusión Y CONCLUSIONES}

La comparación de la difusión de la primavera árabe al movimiento $15 \mathrm{M}$ con la difusión

\footnotetext{
16 Entrevista a Max Berger (26 años), 9/10/2012, Nueva York.

17 Entrevista a Nathan Schneider (edad desconocida, periodista), 19/10/2012, Nueva York.
} 
de este al movimiento Occupy en Estados Unidos muestra que el ítem transmitido y el tipo de vínculos a través de los cuales tuvo lugar la difusión difieren de un proceso al otro. En el primer proceso, los indignados españoles recibieron un fuerte sentido de eficacia de la acción colectiva emprendida por los manifestantes árabes, sobre todo los egipcios. La difusión de este elemento se produjo a través de la publicidad alcanzada por la movilización en los medios de comunicación y las redes sociales y en ausencia de contactos interpersonales. En este sentido, la difusión fue indirecta (Tarrow, 2005). Una vez recibido, el componente agencial de los marcos de acción colectiva se vio influenciado por la permisiva respuesta de las autoridades y la esperanza de cambio alentada por las dimensiones y el apoyo alcanzados por la propia movilización.

En cualquier caso, la difusión de Egipto a España no solo fue ideacional (Givan, Roberts y Soule, 2010), en relación con el componente agencial de los marcos de acción colectiva. También incluyó formas de acción, aunque en este caso los indignados solo recibieron sus contornos generales. Los activistas españoles replicaron la forma general de la protesta egipcia: la ocupación de la plaza central de la ciudad con cierta vocación de permanencia. De nuevo, la difusión tuvo lugar a través de los medios de comunicación (viejos y nuevos). Sin embargo, más allá de los contornos de esta modalidad de protesta (Patel, 2013), la ocupación de Tahrir y las decenas de ocupaciones de las plazas españolas fueron en buena medida diferentes. Las diferencias se explican en parte en referencia a la contienda local y los procesos de aprendizaje colectivo asociados a las experiencias derivadas de movilizaciones sociales recientes (Romanos, 2013). La comparación de este proceso particular de difusión con el que tuvo lugar entre España y Estados Unidos sugiere que estas diferencias también tienen que ver con la ausencia de contactos interpersonales en el proceso de co- municación de elementos más conductuales (Givan, Roberts y Soule, 2010).

El análisis de la difusión del 15M a OWS subraya la transmisión de innovaciones relacionadas con formas de acción y de organización. Los activistas estadounidenses recibieron una forma de inclusividad organizativa hasta cierto punto novedosa, orientada hacia los potenciales participantes en el movimiento y hacia la transformación del espacio público en un espacio abierto a la empatía. Otros elementos también difundidos hacen referencia a la forma de organizar la deliberación, el espacio y la actividad en la acampada para convertirla en un espacio operativo capaz de atraer a potenciales seguidores y de mantener activos a los participantes en la creación de redes y proyectos. Estos elementos se difundieron con la ayuda de los emigrantes españoles residentes en Nueva York que participaron en la organización previa y el desarrollo de OWS.

La comparación de estos dos procesos de difusión ayuda a avanzar en la comprensión del fenómeno de la difusión transnacional de la protesta de diferentes maneras. En primer lugar, la comparación aporta elementos con los que ayudar a responder hasta qué punto un determinado tipo de vínculo es más proclive a la difusión de un determinado elemento (Soule, 2004). La difusión de la primavera árabe en el $15 \mathrm{M}$ no fue solo ideacional, pero la transmisión de elementos conductuales fue limitada. La difusión del 15M en OWS no solo tuvo lugar a través de la actuación de mediadores, en este caso los emigrantes españoles. También se apoyó en los medios de comunicación. Pero precisamente los contactos interpersonales permitieron la transmisión de innovaciones conductuales hasta cierto punto complejas. La comparación de los dos procesos sugiere una relación entre el carácter y los mecanismos de difusión a modo de hipótesis: los elementos ideacionales se difunden fácilmente a través de canales indirectos mientras que las innovaciones conductuales precisan contactos interperso- 
nales para ser difundidas adecuadamente. Los hallazgos de la investigación permiten pensar que el componente agencial de los marcos de acción colectiva se puede transmitir en ausencia de contactos directos entre los transmisores y receptores de la difusión, o de alguien que los ponga en contacto, mientras que otros ítems más complejos relacionados con la organización de los movimientos sociales y el desarrollo de repertorios de acción colectiva necesitan precisamente de esos vínculos interpersonales. Investigaciones futuras deberán confirmar hasta qué punto esa dinámica se observa también en otros procesos de difusión. De confirmarse, la hipótesis plantearía una crítica a los trabajos que enfatizan el papel de los nuevos medios de comunicación en la difusión de los movimientos sociales y la protesta (Tremayne, 2014; Vasi y Suh, 2012; Castells, 2012; Ayres, 1999).

El análisis comparado de los dos procesos también permite avanzar en el debate de otras cuestiones recogidas recientemente por Soule (2013), entre ellas: i) hasta qué punto los mecanismos y procesos de difusión son los mismos en relación con innovaciones ideacionales y conductuales; ii) hasta qué punto estas innovaciones se transmiten con mayor o menor grado de adaptación; y iii) qué efectividad tienen los modelos directos e indirectos para difundir los movimientos sociales. La afinidad sugerida anteriormente entre, por un lado, canales indirectos y elementos ideacionales $y$, por el otro, la presencia de contactos interpersonales y la difusión de elementos conductuales parece estar relacionada con la efectividad de los distintos canales para la difusión de elementos de uno y otro tipo. Los activistas aprenden acerca de formas de acción y organización aplicadas en otros países leyendo sobre las mismas, o viéndolas en Youtube. Es lo que sucedió con la difusión de las ocupaciones, que viajaron de Egipto a España y Estados Unidos. Sin embargo, los hallazgos de esta investigación permiten pensar que la difusión solo fue así en relación con la forma general de esa ocupación. La comparación de los dos procesos sugiere que los contenidos específicos de la ocupación -cómo ocupar y cómo dar sentido a la ocupaciónno se difundieron en el primer proceso sino en el segundo, precisamente a través de la intervención de terceros. Sin duda el volumen de literatura y vídeos disponibles prácticamente al instante fueron relevantes para la difusión transnacional de la protesta, pero el análisis del segundo proceso de difusión sugiere que la participación de mediadores resultó eficaz para difundir conocimientos y técnicas específicos y seguramente más difíciles de aprehender sin la presencia de activistas que los explicaran en detalle y abogaran por su utilización.

Si las experiencias locales fueron importantes a la hora de poner en marcha la ocupación en España (Romanos, 2013), ¿por qué no debería ocurrir lo mismo en Nueva York? Sin duda fue así, y de ahí precisamente la importancia de los emigrantes españoles; emigrantes que Ilevaban tiempo residiendo en la ciudad y que por lo tanto conocían relativamente bien el entorno. La protesta local influyó en la recepción de las innovaciones, que fueron adoptadas con cierto grado de adaptación. Los emigrantes españoles tuvieron que hacer frente precisamente a problemas derivados de la traducción y adaptación de algunas innovaciones. Pero lo que cambia aquí es la disponibilidad y la involucración de los activistas transnacionales - en este caso inmigrantes españoles - en el proceso de difusión. Los testimonios recogidos en esta investigación sugieren que no hubo una involucración semejante de emigrantes egipcios en el primer proceso. Al mismo tiempo, la acción de los emigrantes españoles en Nueva York se produjo en un contexto de alta movilización que atrajo a mucha gente nueva, sin experiencias previas en el campo de los movimientos sociales y que, por ende, seguramente opuso menos resistencia a las propuestas venidas de fue- 
ra, por lo que el siempre presente proceso de adaptación cultural fue relativamente menos intenso que en otros casos de difusión (Wood, 2012; Givan, Roberts y Soule, 2010).

\section{Bibliografía}

Álvarez, Klaudia et al. (2011). Nosotros, los indignados. Barcelona: Destino.

Ayres, J. M. (1999). «From the Streets to the Internet: The Cyber-Diffusion of Contention". Annals of the American Academy of Political and Social Science, 566: 132-143.

Castells, Manuel (2012). Redes de indignación y esperanza. Madrid: Alianza.

Chabot, Sean (2010). «Dialogue Matters: Beyond the Transmission Model of Transnational Difussion between Social Movements». En: Givan, R. K.; Roberts, K. M. y Soule, S. A. (eds.). The Diffusion of Social Movements. Cambridge: Cambridge University Press.

Della Porta, Donatella (2005). «Making the Polis: Social Forums and Democracy in the Global Justice Movement». Mobilization, 10(1): 73-94.

Della Porta, Donatella y Diani, Mario (2011). Los Movimientos Sociales. Madrid: CIS-UCM.

Diani, Mario (2011). «Networks and Internet into Perspective». Swiss Political Science Review, 17(4): 469-474.

Doerr, Nicole (2008). «Deliberative Discussion, Language, and Efficiency in the WSF Process». Mobilization, 13(4): 395-410.

Flesher Fominaya, Cristina (2014). Social Movements and Globalization. New York: Palgrave.

Gamson, William (2011). «Arab Spring, Israeli Summer, and the Process of Cognitive Liberation». Swiss Political Science Review, 17(4): 463-468.

Ghonim, W. (2012). Revolution 2.0: A Memoir. London: Fourth State.

Gitlin, Todd (2012). Occupy Nation. New York: Harper Collins.

Givan, Rebecca Kolins; Roberts, Kenneth M. y Soule, Sarah A. (2010). The Diffusion of Social Movements. Cambridge: Cambridge University Press.

Goldstone, Jack (2011). "Cross-class Coalitions and the Making of the Arab Revolts of 2011». Swiss Political Science Review, 17(4): 457-462.
Gordon, Uri (2012). «Israel's "Tent Protests": The Chilling Effect of Nationalism». Social Movement Studies, 11(3-4): 349-355.

Graeber, David (2011). «On Playing by the Rules: The Strange Success of \#OccupyWallStreet». Naked Capitalism, (en línea). http://www.nakedcapitalism.com/2011/10/david-graeber-on-playing-bythe-rules- --the-strange-success-of-occupywall-street.html, último acceso 30 de noviembre de 2012.

Hammond, John (2013). «The Significance of Space in Occupy Wall Street». Interface, 5(2): 499-524.

Kerton, Sarah (2012). «Tahrir, Here? The Influence of the Arab Uprisings on the Emergence of Occupy». Social Movement Studies, 11(3-4): 302-308.

Kroll, Andy (2011). «How Occupy Wall Street Really Got Started». En: van Gelder, S. (ed.). This Changes Everything. San Francisco, California: Berrett-Koehler Publishers.

Kurzman, Charles (2012). "The Arab Spring Uncoiled». Mobilization, 17(4): 377-390.

Lawrence, Jeffrey (2013). «The International Roots of the $99 \%$ and the "Politics of anyone" $. I C-R e-$ vista Científica de Información y Comunicación, 10: 53-72.

Muñoz, Alba (2011). «Del síndrome Wikileaks a la democracia 2.0. Las redes sociales y el 15-M"». En: Antentas, J. M. et al. (eds.). Las Voces del 15-M. Barcelona: Los libros del lince.

Naidu, Suresh (2011). "My Trajectory with Occupy». En: Schiffrin, A. y Kircher-Allen, E. (eds.). From Cairo to Wall Street. New York: The New Press.

Patel, David S. (2013). «Roundabouts and Revolutions: Public Squares, Coordination, and the Diffusion of the Arab Uprisings". Paper inédito.

Rogers, E. M. (1995). Diffusion of Innovations. $4^{\text {a }}$ ed. New York: Free Press.

Romanos, Eduardo (2011a). «El 15M y la democracia de los movimientos sociales». Books and Ideas, 18 de octubre.

Romanos, Eduardo (2011b). «Emociones, identidad y represión: el activismo anarquista durante el franquismo». Revista Española de Investigaciones Sociológicas, 134: 87-106.

Romanos, Eduardo (2013). "Collective Learning Processes within Social Movements: Some Insights into the Spanish 15M/Indignados Movement». En: Flesher Fominaya, C. y Cox, L. (eds.). Understanding European Movements. London: Routledge. 
Sitrin, Marina y Azzellini, Dario (2012). Occupying Language. New York: Occupied Media Pamphlet Series.

Snow, David. A. y Benford, Robert. D. (1992). «Master Frames and Cycles of Protest». En: Morris, A. y Mueller, C. (eds.). Frontiers of Social Movement Theory. New Haven, Connecticut: Yale University Press.

Soule, Sarah A. (2004). «Diffusion Processes within and across Movements». En: Snow, D. A.; Soule, S. A. y Kriesi, H. (eds.). The Blackwell Companion to Social Movements. Malden, Massachusetts: Blackwell.

Soule, Sarah A. (2013). «Diffusion and Scale Shift». En: Snow, D. A. et al. (eds.). The Wiley-Blackwell Encyclopedia of Social and Political Movements. Malden, Massachusetts: Blackwell.

Tarrow, Sidney (1989). Democracy and Disorder: Protest and Politics in Italy, 1965-1974. New York: Oxford University Press.

Tarrow, Sidney (2005). The New Transnational Activism. Cambridge: Cambridge University Press.

Tejerina, Benjamín et al. (2013). «From Indignation to Occupation: A New Wave of Global Mobilization». Current Sociology, 61(4): 377-392.
Tilly, Charles y Tarrow, Sidney (2007). Contentious Politics. Boulder, Colorado: Paradigm.

Toret, Javier (2012). «Una mirada tecnopolítica sobre los primeros días del \#15M». En: Alcazan et al. (eds.). Tecnopolítica, internet y r-evoluciones. Barcelona: Icaria.

Tremayne, M. (2014). «Anatomy of Protest in the Digital Era: A Network Analysis of Twitter and Occupy Wall Street». Social Movement Studies, 13(1): 110-126.

Vasi, Ion B. y Suh, Chan S. (2012). «Protest in the Internet Age: Public Attention, Social Media, and the Spread of the "Occupy" Movement in the United States". Paper presented at the American Sociological Association Annual Conference, Denver, Colorado.

Voulgarelis, Antonis (2012). «Nights in Syntagma Square». En: Schiffrin, A. y Kircher-Allen, E. (eds.). From Cairo to Wall Street. New York: The New Press.

Walsh-Russo, C. (2014). «Diffusion of Protest». Sociology Compass, 8(1): 31-42.

Wood, Lesley J. (2012). Direct Action, Deliberation, and Diffusion: Collective Action after the WTO Protests in Seattle. Cambridge: Cambridge University Press. 


\title{
From Tahrir to Puerta del Sol to Wall Street: The Transnational Diffusion of Social Movements in Comparative Perspective
}

\author{
De Tahrir a Wall Street por la Puerta del Sol: la difusión \\ transnacional de los movimientos sociales en perspectiva comparada
}

Eduardo Romanos

\section{Key words}

Comparative Analysis

- Diffusion of

Innovations

- Public Space

- Protest Movements

- Social Movements

\section{Palabras clave}

Análisis comparativo

- Difusión de

innovaciones

- Espacio público

- Movimientos de

protesta

- Movimientos sociales

\begin{abstract}
This article examines social movement diffusion through a comparison between two processes: diffusion from the Arab Spring to the Spanish indignados, and from the latter to Occupy Wall Street. The comparison shows that the content and the channel of diffusion differ from one process to the other. The Spanish indignados received a sense of collective efficacy from the Arab Spring essentially through indirect means. In the second process, the mediation of third parties facilitated the diffusion of more practical knowledge related to new forms of inclusivity and the occupation of public space. The comparison of these two processes suggests that ideational elements are easily transmitted through indirect channels, whereas behavioural innovations require interpersonal contact to be properly transmitted.
\end{abstract}

\section{Resumen}

Este artículo compara la difusión de la primavera árabe al movimiento $15 \mathrm{M}$ con la de este último a Occupy Wall Street. La comparación muestra que el contenido y el canal de la difusión fueron diferentes en uno y otro proceso. Los indignados españoles recibieron de la primavera árabe un sentido de eficacia de la acción colectiva a través fundamentalmente de medios indirectos. En el segundo proceso, la mediación de terceros facilitó la difusión de conocimientos más prácticos y complejos relacionados con nuevas formas de inclusividad y la ocupación del espacio público. La comparación sugiere que los elementos ideacionales se difunden fácilmente a través de canales indirectos mientras que las innovaciones conductuales precisan contactos personales para ser difundidas adecuadamente.

\section{Citation}

Romanos, Eduardo (2016). "From Tahrir to Puerta del Sol to Wall Street: The Transnational Diffusion of Social Movements in Comparative Perspective". Revista Española de Investigaciones

Sociológicas, 154: 103-118.

(http://dx.doi.org/10.5477/cis/reis.154.103) 


\section{INTRODUCTION ${ }^{1}$}

It is commonplace in the literature on the recent transnational wave of protests to recognise a connection among the different protests that have taken place at the local level. Observers and participants agree that certain protest movements have influenced others. Thus, Tunisia and Iceland "have become reference points for the social movements that shook the political order in the Arab world and challenged European and American political institutions" (Castells, 2012: 37). The Arab Spring in turn influenced the uprising of the Spanish indignados (Flesher Fominaya, 2014; Tejerina et al., 2013; Castells, 2012), while the movement that began in Madrid "prompted the rest of Europe to participate, causing a domino effect throughout the rest of the continent and of course across the Atlantic, with the Occupy Wall Street movement" (Voulgarelis, 2012: 171-172). Occupy Wall Street drew from these sources, as well as from others closer by, such as the occupation in Madison, which in turn were boosted, along with countless other new occupations in the United States, by the protests in New York (Kerton, 2012; Gitlin, 2012; Kroll, 2011; Hammond, 2013).

However, while this influence has been recognised, there is still a lack of empirical studies inquiring into the dynamics of diffusion involved in the spread of protest from place to place. This article aims to help fill this gap based on a comparison between

\footnotetext{
1 This article is part of the project, CSO2013-41035-P, funded by the Ministry of Economy and Competitiveness. I presented a preliminary version of this article in a series of conferences in Montreal, Barcelona, Madrid, El Escorial and Paris. I want to thank the participants in these meetings for their interesting comments, as well as Jack Hammond, Cristina Flesher Fominaya and the two anonymous reviewers of this journal. I would also like to thank David Siddhartha Patel, Ion Bogdan Vasi and Chan S. Suh for sending me their manuscripts and allowing me to cite them here, and Sydney Tarrow for his brokerage with these authors.
}

two diffusion processes within the recent wave of protests: the diffusion of the Arab Spring to the Spanish indignados movement (also known as the 15M movement) and from this movement to Occupy Wall Street (OWS) in the United States. The analysis encompasses the nine month period between the beginning of the Arab Spring (December 2010) and the emergence of OWS (September 2011), with the emergence of the $15 \mathrm{M}$ movement in Spain in between (May 2011); nine months of intense mobilisation that we can identify with the beginning of various cycles of protest at the local level, in this case in North Africa and the Middle East, Spain and the United States.

The concept of diffusion has received a great deal of attention in the literature on social movements (Walsh-Russo, 2014; Givan, Roberts and Soule, 2010). When researchers in the field speak of diffusion they mean "some element of a social movement (e.g. tactic, frame, ideology, protest, repertoire, campaign) is spreading across some set of actors (e.g. organisations, networks, groups, people, communities, states) in a social system either through direct or indirect networks of communication" (Soule, 2013). Narrower definitions suggest that the element to be transmitted must be an innovation (Rogers, 1995; Soule, 2004; Givan, Roberts and Soule, 2010). All diffusion processes consist in any case of four basic elements: a transmitter, an adopter, an item to be diffused, and a channel along which the item may be transmitted. The comparison included in this article focuses on the last two in order to better understand to what extent a certain type of channel is more likely to facilitate the diffusion of a specific item. Soule (2004) posed this question in her review of the literature on social movement diffusion and encouraged researchers to produce comparisons that would help answer it.

Social movement scholars abandoned a while ago analyses that understood diffusion to be a product of contagion, imitation or ra- 
tional choice and developed new approaches "that see adopters and rejecters of innovations as active participants (both individual and groups) engaged in meaningful social interaction" (Wood, 2012: 8). Theoretical refinement has produced different typologies. Recently, Givan, Roberts and Soule (2010) have focused on the character of the item to be diffused in order to differentiate two types of diffusion: behavioural and ideational. The former refers to the transmission of tactics, forms of organisation or repertoires of action, while the latter consists of the transmission of collective action frames. Tarrow (2005: cap. 6), in his highly influential study in the area, distinguished between three types of diffusion according to the mechanisms underlying each of them: direct (or relational) diffusion, which depends on the interpersonal ties between the initiators and the adopters of an innovation; indirect (or non-relational) diffusion, which relies on impersonal ties mainly through the media; and mediated diffusion, which relies on the intermediation of third parties who act as translators or brokers among actors, who might otherwise not have contact with each other or not recognise their mutual interests. These typologies and their implications will be discussed in the comparative analysis of the two diffusion processes constituting the case studies in this paper.

This article is organised as follows: after a brief presentation of the three protest movements (the Arab Spring, 15M and OWS), the two diffusion processes are analysed separately (from the Arab Spring to $15 \mathrm{M}$, and from $15 \mathrm{M}$ to OWS) in order to compare them. This is followed by a final section of discussion and conclusions that aims to advance the line of work posed by Soule. The sources analysed include, in addition to documents, web pages and secondary literature, 30 semi-structured interviews carried out individually with activists from the $15 \mathrm{M}$ and OWS movements in two phases: 10 in Madrid and Barcelona between October 2011 and August 2012 and 20 in New York between September and October 2012 (of those interviewed, $60 \%$ were men and $40 \%$ were women). It is important to stress that the main object of this research was not the protests themselves, but rather the diffusion processes between them, and concretely, the content and channels employed in two of these processes. The interviews carried out in Spain were aimed at finding out what was diffused from the Arab Spring and through which channels. In this regard, I asked the $15 \mathrm{M}$ activists what previous protests had influenced them (if there were any), which specific aspects of these protests had gotten their attention, and how they had known about them. The interviews with the North American activists in OWS (7 of the 20 interviewed in New York) included these same questions. The fieldwork in New York included another 13 interviews with Spanish immigrats who participated in the emergence of OWS. I asked them, among other questions, what aspects of $15 \mathrm{M}$ had gotten their attention, how had they known about these aspects, and how they had transmitted them to OWS, if they did so. The role played by these transnational activists is indeed a fundamental difference between the two diffusion processes, which in turn helps us to understand differences in the innovations diffused in one case and the other. As will be discussed subsequently, there was no interpersonal contact between indignados and activists or immigrants from North Africa and the Middle East that was strong enough to support the case for direct or mediated diffusion from the Arab Spring to $15 \mathrm{M}$. In this case, diffusion took place though other channels (indirect). It also had other content (more ideational).

\section{MOBILIZATION WITHIN THE NEW WAVE OF PRotests: Arab SpRING, 15M AND OWS}

The Arab Spring is a paradigmatic example of the "streams of contention" defined by Tilly and Tarrow (2007: 211), in which one of 
the contenders is the government, though in this case it was a series of govermments. Among the emblematic places in the Arab Spring protest was Tahrir Square in Cairo. Egyptian demonstrators occupied the square on January 25, 2011. The Facebook group "We are all Khaled Said", created by Wael Ghonim (2012) in memory of a young activist beaten to death by the police months before in Alexandria, played an important role in launching the protest. For several days, various protest marches arrived at Tahrir Square from different points in the city, while the Square itself was turning into a huge tent city (Patel, 2013). The square was also the site of fierce confrontations between opponents and defenders of the regime, with the latter supported by the police. The government ordered the army to intervene, but they took an ambiguous position, until finally deciding to support the regime's opponents, which was the final blow to president Mubarak, who was forced to resign after 18 days of protest and nearly 30 years in power.

A few months later, on the 15 of May, 2011 across the Mediterranean, protest marches called by the Real Democracy Now platform, under the slogan, "We are not products in the hands of bankers and politicians", were able to mobilize tens of thousands of people all over Spain. In Madrid, some of the marchers decided to continue the protest by blocking traffic in the centre of the city with a sit-in. After confrontations with the police, which ended with numerous arrests, a group of 40 persons remained in Puerta del Sol, for among other reasons, in solidarity with those who had been arrested. From this gathering, an assembly arose with the idea of creating and maintaining a permanent camp (Romanos, 2013). With this, was born the acampadasol, which started to grow around the emergence of various committees dedicated to maintaining the camp and the logistics of the assembly process, as well as various work groups dedicated to generating discourse related to the emerging protest movement. Support for the protest grew both on the Internet and at Puerta del Sol and other squares in Spain, where other camps were being set up. The Electoral Board of Madrid prohibited protests during the "day of reflection" prior to the municipal and regional elections on 22 May. Around 25,000 people defied the decision in a massive act of civil disobedience. The camp was disbanded on the 12th of June after long internal discussions and pressure from the authorities.

Four days later a group of New Yorkers camped out in front of City Hall to protest the cuts and austerity policies of Michael Bloomberg. The so-called Bloombergville lasted three weeks, disbanding after the approval of a new municipal budget. Some of those who had been camping there went to a meeting afterwards at 16 Beaver Street, an artivist space near Wall Street where Spanish, Greek and Egyptian immigrants shared their impressions about the protests that had been taking place in their countries of origin and how to extend this across the Atlantic. Some of those present went to a protest on August 2nd at Bowling Green Park, the Charging Bull square in Lower Manhattan, from which emerged an assembly that would afterwards be held on Saturday afternoons in Tompkins Square Park in the East Village (Kroll, 2011) under the name of the New York City General Assembly (NYCGA). Around 50 or 60 people would usually attend the meetings, over half of them immigrants (Lawrence, 2013). By then, Adbusters had already launched its call to fill Manhattan with "tents, kitchens [and] peaceful barricades and to occupy Wall Street for months" starting on September 17th. That day, thousands of demonstrators marched through the financial district of New York and ended up setting up a camp in Zuccotti Park. Its form resembled the camps of Tahrir and Puerta del Sol, although on a smaller scale. Inside the camp an assembly was held every day facilitated in part by those who had participated in the NYC- 
GA. The occupiers created a powerful slogan - We are the 99\% - although media attention came largely because of the disproportionate police response, disseminated through social media networks. Meanwhile, occupations started happening all over the country (Vasi and Suh, 2012). On the 15th of October, the Occupy movement participated in the huge Global Day of Action launched by the Spanish indignados. One month later, the police expelled the occupiers from Zuccotti Park.

\section{The diffusion of the Arab SPRING TO THE 15M MOVEMENT IN SPAIN}

\section{The differential diffusion of collective action frames}

In a brief article, William Gamson (2011) discussed the influence of the Arab Spring on the protests that spread to Israel in the summer of 2011. Initiated on the 14th of July, the protests in favour of a new social contract in Israel culminated on September 3rd with the largest demonstration in the history of the country (Gordon, 2012). Gamson analysed the connections between these protests based on the concept of collective action frames, that is, "action oriented sets of beliefs and meanings that inspire and legitimate social movement activities and campaigns" (Snow and Benford, 1992; cited in Gamson, 2011). The concept of collective action frames is composed of three elements: the injustice component, which refers to the moral indignation expressed in the form of political consciousness; the agency component, which refers to the awareness that it is possible to alter conditions or policies through collective action, and the identity component, which refers to the process of defining a "we" in opposition to a "they" who have different interests and values. According to Gamson (2011: 464), the influence that knowledge about collective action in one given country might have in other countries refers almost exclusively to the agency component, while the components of injustice and identity are almost entirely based on local conditions. In the Arab Spring and the protests in Israel, the sense of injustice was based on different grievances, and the cleavages that the construction of an inclusive "we" aimed at breaking down were also different. Nevertheless, the sense of collective efficacy linked to the campaign of non-violent protest and civil disobedience travelled from Tunisia to Egypt first, and then from Egypt to Israel (Gamson, 2011).

\section{The agency component across the Mediterranean}

Did the Arab Spring influence the $15 \mathrm{M}$ movement in a similar way? Let us look at the three components of collective action frames one by one. Among the economic and political grievances underlying the popular uprisings in North Africa and the Middle East, those that stand out are poverty, the high levels of unemployment, police brutality, limited freedom of speech, the arbitrariness of the state and government corruption (Kurzman, 2012). The protests that the Spanish indignados movement initiated made reference to some of these forms of injustice; for example, the privileges of the political class and unemployment. But for the most part, the problems focused on were different: the lack of control of the banks, access to housing, deteriorating public services, taxation, representative democracy and military spending (Toret, 2012: 55). In the camp set up in Madrid, discussions focused on certain issues, which greatly differed from the grievances that sparked the Arab Spring, such as reform of the electoral law, effective separation of powers and political accountability. ${ }^{2}$

\footnotetext{
2 http://madrid.tomalaplaza.net/2011/05/26/acampadasol-consensua-cuatro-lineas-de-debate/
} 
Regarding the identity component, Gamson (2011) suggested that in the beginning of the Arab Spring some organisations such as the Muslim Brotherhood in Egypt made a deliberate effort not to frame the uprising in Islamist terms, which facilitated understanding with disadvantaged sectors, regardless of the intensity or nature of their religious convictions. Goldstone (2011) also noted the importance of cross-class and cross-regional coalitions in Tunisia, Egypt and Libya. In Spain, as happens in general in the early phases of protest cycles (Tarrow, 1989), the activists also chose to construct an inclusive "we", although here the process was to a large degree different: those held responsible were clearly identified (the political and economic elites in coalition to defend their interests) and the drafting of general demands helped to bring about the participation of a large part of the population. The possible reticence of individuals without previous experience in social movements was countered by a series of elements that encouraged participation; for example, holding open assemblies, the absence of partisan symbols, the creation of respect committees and anonymity (on the Internet and in the squares).

While the processes of constructing a sense of injustice and a collective identity in the $15 \mathrm{M}$ movement were anchored in local conditions, the agency component of the collective action frames seems to have been strongly influenced by the Arab Spring, as was the case in Israel. This was recognised by Javier Toret (2012: 55), one of the organisers of the $15 \mathrm{M}$ protest:

The protest aimed at creating links with emerging movements in Europe... But what really sparked the call to protest was the "Arab spring". The contagious force of those revolts inspired many people who started to believe that rebellion was possible.

The influence of Tahrir was also felt in the camps set up after the 15 of May demonstration. Asked what he thought was going to happen after the first night in Puerta del Sol, one of those camping there replied:

What happened in Egypt, people out on the streets and occupying squares... we believed that could happen [in Spain]. Obviously we didn't expect it could reach the same level...but we did think it was possible... For me, that was really a powerful thought. ${ }^{3}$

After Mubarak's fall, the Spanish activists were aware of the effectiveness of collective action that made skilful use of social networks and that simultaneously had a strong presence in the squares. It was commonplace among the indignados to think that without Facebook, Twitter and Youtube the Arab Spring would not have been possible. Beyond the real part played by these social networks in that protest movement (Diani, 2011), the members of Democracia Real Ya [Real Democracy Now] saw in the Egyptian twitter users the enormous potential of using technology for political purposes, not so much for transmitting information but as a form of of "interactive organisation" (Muñoz, 2011: 42). This use was not new, but it did seem to be so in its mobilising capacity. Moreover, the occupation of Tahrir became a symbol that the indignados would later reproduce in their own way in Puerta del Sol. Ahead of the Israelis and Americans, the Spanish were the first to take to the streets and set up tents en masse, occupying the main squares and parks of their towns and cities, replicating the techniques of non-violent civil disobedience they had seen in Tahrir.

\section{How did agency travel and bear fruit?}

The Spanish indignados received information about the collective action in Egypt and other countries in the region through media cov-

\footnotetext{
3 Interview with Miguel (29 years old, doctoral student in theoretical physics) March 6, 2012, Madrid.
} 
erage and especially through online social networks. The testimonies of activists collected in the interviews suggest that there was no or very little interpersonal contact. This does not mean that the knowledge the Spanish indignados had about the Arab Spring was superficial. For four months, the organisers of the Democracia Real Ya campaign followed the Egyptian twitter users and how they used new technologies (Muñoz, 2011). As previously noted, the social networks were used precisely to promote a sense of connection that potential activists did not find in conventional media. In this regard, Carolina, hacker and participant in the acampadasol points out how:

The way they used technology, how they kept Twitter, Youtube active, made a positive impression on me and I said to myself: "they are speaking directly to the people. This is not mediatised, not even by alternative media, but all of a sudden I am seeing something, I don't know [exactly] what's going on, but I get it". 4

Gamson (2011: 467) argues that once the sense of collective efficacy is transmitted, it is heavily influenced by the response of authorities. If the measures of control fail, the agency component increases. This is what happened in Tahrir, where the army refused to repress the demonstrators; it also happened in Puerta del Sol, where the police dislodged the camp on the second night, but were unable to cope with the public's reaction, and finally the authorities decided against the use of force, which in a certain way strengthened the protest. However, not only do the influence of other mobilisations and the failure of social control measures increase the sense of collective agency, it is also the protest itself, which helps to create a sense of hope in which, maybe not every-

\footnotetext{
4 Video conference interview with Carolina (April 8, 2012).
}

thing, but many things are possible (Romanos, 2011b). In the case of the Spanish indignados, the early success of their protest in terms of participation and the repercussions in the media led some to believe that "there would be uprisings in all the squares in the world during that first week". 5 The sense of collective agency grew, and the indignados went from being "anaesthetized" or "paralysed" to participating in collective action (Álvarez et al., 2011): hence, the plaque in Puerta del Sol which reads, "We were asleep; we woke up. Square occupied".

\section{THE DIFFUSION FROM 15M TO OWS}

Having analysed diffusion from Egypt to Spain, this section will now look at diffusion from Spain to the United States. Selecting and studying these two processes separately does not mean that they did not overlap; nor does it mean that there have not also been other processes of diffusion within the recent transnational wave of protests. The popular uprising of the Egyptians undoubtedly had an influence on the emergence of Occupy Wall Street. In fact, the call from Adbusters began by asking: "Are you ready for a Tahrir moment?" The agency component travelled from Egypt to New York, propelled by the visibility that the Spanish indignados achieved internationally. the diffusion of the Arab Spring to the Spanish indignados seems to have been in some aspects different from the diffusion from the indignados to Occupy Wall street.

\section{Spanish immigrants in New York}

An important difference between the two processes of diffusion lies in the role played by transnational activists and in particular by Spanish emigrants residing in New York.

\footnotetext{
5 Stephane M. Grueso's interview with Miguel. Available at http://madrid.15m.cc/2011/12/conversaciones15mcc-miguel-arana.html> (access: 15/2/2013).
} 
Months before the emergence of OWS, Spanish immigrants created an activist network in the city. On 21 May, 2011, several hundred people demonstrated in Washington Square in conjunction with the protests taking place the same day in Puerta del Sol in Madrid and other Spanish cities. The demonstration was called by the Facebook group, Democracia Real Ya-New York, created after the success of the 15May demonstrations in Spain. The group grew rapidly on online, and approximately sixty of its members met each other in person for the first time at Columbia University on 19 May. This location was no accident. A large number of the Spanish immigrants mobilised were university students and researchers. Some of them understood the movement taking place in squares all over Spain to be part of a much broader wave of protests related to global problems, and they got organised in order to extend the protest to their host country, seeking the attention of local media and groups. Over time, they formed an affinity group participating very actively in the protests preceding the occupation of Zuccotti Park (Kroll, 2011; Graeber, 2011; Lawrence, 2013). Before the occupation, some of them organised a talk in a book store and activist centre on the Lower East Side to explain what was happening in Spain. The Spanish emigrants were following the protests through conventional media, social networks and interpersonal communication with family and friends participating in them. Some of them also travelled to Spain and were able to participate briefly in the protests there. As will be seen subsequently, these experiences helped them to acquire knowledge that was later transmitted to the new local context.

The talk in the book store was the first step in an effort to connect with local activists. Shortly afterwards, there was a visit to Bloombergville, where the Spanish immigrants had conversations with activists camped there, some of whom received the information about the protests in Spain with a mixture of "incredulity, ignorance and also some degree of enthusiasm." There was apparently greater receptivity at the meeting at 16 Beaver. There, a certain degree of common ground was established, which was later strengthened in the development of the New York City General Assembly (NYCGA), with some of the Spanish activists joining the outreach working group "with the idea that [this] should be a movement not just for activists but for everybody."6

\section{Organisational innovations and problems with translation}

As mentioned previously, inclusiveness was a fundamental value in the $15 \mathrm{M}$ movement. However, in no way it was something new in the field of social movements. We can find forms of organisational inclusiveness in post1968 movements, for example, in the women's movement. Della Porta (2005) notes how the activists in the anti-globalisation movement adopted this principle even more strongly, with it becoming one of the basic features of the deliberative democracy model practised in its networks. This model promotes the inclusion of the enormous diversity of actors forming this "movement of movements" in decision-making. To this end, mechanisms are established, such as the general assembly, in which each person can express his or her opinion.

The indignados movement basically adopted the deliberative democracy model (Romanos, 2011a). However, we can see two aspects of inclusiveness that were somewhat new, aspects that in turn were transmitted to the activists in the United States. First, the inclusiveness promoted by the indigna-

\footnotetext{
6 Interview with Luis Moreno-Caballud (36 years old, assistant professor), October 6, 2012, New York. According to Lawrence (2013), the Spanish, most of whom had graduate degrees, usually represented between $10 \%$ and $20 \%$ of the participants in the meetings of the NYCGA. Most of them did not have prior experience participating in social movements.
} 
dos was not targeted at those already participating in the movement - to establish mechanisms to ensure their inclusion in the decision-making process - but rather at potential participants. Here the square played an important role. One of the novel aspects of $15 \mathrm{M}$ was the way it placed experiments with new concepts of democracy in the centre of public space. In this way, the movement brought practices of deliberative democracy from spaces that were more or less limited (e.g. camps, social forums or social centres) to public squares, where passers-by were invited to join in. This seems to be an important difference with previous movements and mobilisations. As Lawrence (2013) noted, the change in space implies a change in the orientation of the movement; the focus of attention is placed on ordinary people from outside the assembly and not so much on the activities of those already involved in these gatherings. Lawrence himself (2013: 9) was witness to the way that the Spanish emigrants promoted this kind of inclusiveness in the NYCGA:

During the assemblies in Tompkins Square Park, while much of the debate focused on tactical and logistical questions related to the occupation, you would often see [Begoña] Santa-Cecilia [a Spanish artist based in Brooklyn] distributing flyers to curious onlookers who were passing through the park, and she would be talking to them about the rationale of Occupy. The idea was that the assembly had to remain open to the $99 \%$ of the population that it considered to be the actual protagonist of the movement, rather than separating itself as a small revolutionary vanguard.

The second aspect has to do with a less rational and more affective meaning of inclusiveness, one not so much oriented toward the decision-making process, but rather to the transformation of public spaces into a space open to empathy as well. In August of 2011, some indignados were reflecting on the basic features of the movement in Spain, one of which was "INCLUSIVENESS. The power of this movement is in the fact that we are many and we are different [...] The spaces that make us strong, that give us joy and make us powerful, are those which allow each one of us to feel them as our own."7 According to some Spanish emigrants in New York, this concept of inclusiveness, which we could call inclusiveness based on empathy, or empathic inclusiveness, was not encouraged in the NYCGA. The assembly was oriented more toward strategic issues, which in their opinion, could represent problems in terms of opening it up toward people without previous experience participating in social movements:

We didn't see [in the NYCGA] the affective dimension we found in Spain, talking to a person who is going through problems, who is feeling bad... So I decided to mention that in every assembly. If I spoke I would say, "We are not here just to talk things over, or to make plans; we are people with problems and our feelings are involved". I thought that was very important. Besides, you always had the typical ego clashes. And I thought, we thought, that if there wasn't that warm feeling that $15 \mathrm{M}$ had, that empathy, then we were not interested [in the movement].

These were the words of Luis MorenoCaballud, who in answering a question about the response to that message, replied that he was under the impression that those words "were not received with the same incredulity or incomprehension as sometimes was perceived when we talked about Spain."8

However, the process of transmitting this concept of inclusiveness ran into an obstacle that is typical in diffusion processes - translation problems (Doerr, 2008; Chabot, 2010; Wood, 2012). An example of this type of

\footnotetext{
7 http://madrid.tomalaplaza.net/2011/08/12/

8 Interview with Luis Moreno-Caballud, October 6, 2012, New York.
} 
problem occurred when the OWS outreach group tried to develop a message to attract participants to attend the September 17th demonstration being organised by $\mathrm{Ad}$ busters. The message identified a social problem not so much in "the corporate domination of our economy and government" but rather in the effects that domination had over "our lives and communities". When it was read before the assembly, some activists who had participated in social movements in the 1960s criticized the use of what they regarded as hackneyed terms, such as "empowerment". Finally, the assembly decided that the proposal should be reformulated. One witness to the process explained that in general the "emotional" discourse of the Spaniards was quite groundbreaking, but it was a translation from Spanish and provoked criticism. In his opinion, "[the local /American activists] basically rejected the language in that document, but a large part of the message was included". 9 In this regard, transmitters and receivers maintained a kind of communication that modified the form of the message before it was finally adopted (Chabot, 2010). The immigrants contributed to the elaboration of a message that was not adopted until it used a language that made sense to the potential receivers. Once translated into that language, the receivers implemented the innovations, thus completing the diffusion process.

\section{How to occupy and how to give meaning to the occupation}

After September 17th, the Spanish activists continued to promote the opening up of OWS in the way of $15 \mathrm{M}$. In this regard, two initiatives stand out. One was setting up a series of tables to welcome people and inform them about what was happening in Zuccotti Park. This idea came from two activists who had

9 Interview with Jeff Lawrence (28 years old, doctorate degree), October 16, 2012, New York. gone to Madrid and knew about the information tables at acampadasol:

If we hadn't seen that information stand, we would have been clueless... We wouldn't have known how we could help, what we could do...There was a lot of information, and there were people there to tell you about what was happening in the city related to $[15 \mathrm{M}]$. That was our idea. Information is what matters the most. Welcome the people is the most important thing in the square. 10

Isham Christie (27 years old, masters student), US activist first in NYCGA and later in OWS, notes how important it was that the information stands organised by the Spanish immigrants were set up in Zuccotti Park:

It was right after we got really really popular in the media so we had so many people coming down. The people didn't know how to get plugged in. They [the Spaniards] were really helpful in getting a bunch of tables to like say "these are the different working groups". And that was a huge contribution, but it's not gonna be written in the pages of the history, but the people had a place to go and participate. ${ }^{11}$

The second initiative was the organization of open forums: "a discussion in Liberty Square where a volunteer speaker offers a brief presentation that is relevant to the protest, followed by an open discussion".12 Naomi Klein came, Slavoj Žižek and others. Following the example of what they had seen in the $15 \mathrm{M}$ movement, the Spanish emigrants wanted to move beyond the occupation, proposing actions that would give it meaning: "The emphasis was on occupying but no-

\footnotetext{
10 Interview with Begoña Santa-Cecilia, October 4, 2012, New York.

11 Interview with Isham Christie, November, 10, 2012, New York.

12 http://www.nycga.net/groups/education-and-empowerment/docs/minutes---empowerment-and-education-working-group-meeting---12211---60-wall-st
} 
body seemed to understand that the square had to be used for something". 13 Judging by the testimonies of US activists, it seems that their efforts were not in vain:

The idea of the occupation staying active, demanding that people work, not in an abusive way, but from a sense of working collectively, in committees; the idea that there was a lot to do. That this wasn't just a sleepover, but a very active occupation. That feeling came from the Spaniards. ${ }^{14}$

Later, the group running the open forums would work toward connecting the activity in the square with community groups and activists working in other parts of New York City. After the Zuccotti Park occupation eviction, the group changed its name to Making Worlds, and its members were still mostly Spaniards.

The Spanish emigrants in New York also helped transmit other innovations by distributing documents created by the Spanish indignados. The most important was Cómo Cocinar una Revolución Noviolenta [How to Cook Up a Non-Violent Revolution], which explained the internal organisation (in committees, work groups and general assemblies) and the horizontal process of decision making in the squares. It also included other information, such as an explanation of who the indignados were and why they were protesting, in addition to an organigram-map of acampadasol. Its authors were the members of the World Extension Team, a commission set up in the first days of the camp in Madrid, aimed at helping to coordinate a protest movement on the international level. The Spaniards translated and distributed the document in NYCGA and OWS. It was also posted on various web pages, among them

\footnotetext{
13 Interview with Vicente Rubio (32 years old, doctoral student), September 29, 2012, New York.

14 Interview with Justin Wedes (26 years old, educator), October 6, 2012, New York.
}

takethesquare.net. However, judging by the testimonies collected in the interviews, its reception in the United States was facilitated by the interpersonal contacts made by the Spanish emigrants and other transnational activists: US activists who had participated in the movement in Spain and who later participated in the rise and development of OWS. According to local activists and observers, these materials were important in Zuccotti Park, especially in relation to the functioning of the general assembly and the organisation of the space in the camp. ${ }^{15}$

\section{Transnational diffusion and global contention}

In any case, the practices disseminated by the Spanish emigrants were not the only source of democratic organisation in Zuccotti Park. Other activists with experiences in horizontalism, for example, from the antiglobalisation movement or the protests in Argentina in 2001, also participated in OWS and served as an inspiration (Sitrin and Azzellini, 2012; Naidu, 2011). This confluence of different experiences suggests the importance of the existence of a global field of contention when analysing specific processes of transnational diffusion. In addition to specific innovations learned from a particular mobilisation, activists were influenced by a broader global imaginary of contentious discourses and practices. In any case, the reference to other experiences of horizontalism helps us to understand certain differences between the deliberative processes in Zuccotti Park and the Spanish squares. For example, the "progressive stack" designed to ensure that marginalised groups get the opportunity to speak was not formalised in Spain, and consensus in Spain had to be complete, which undoubtedly blocked the approval of many proposals. In New York, on

15 Interview with Isham Christie, November 10, 2012, New York. 
the other hand, a modified form of consensus that accepted small discrepancies was the practice. Other differences had to do with the political context and specifically with the policing of protest. In this regard, the prohibition of any type of sound equipment in Zuccotti Park meant that they had to use the socalled "human microphone", with all that implied: more participation and closeness but at the same time, less fluency and sophistication in developing discussions.

Besides the Spanish emigrants and the US activists who travelled to Spain, there were other transnational activists who seemed to have served as an important link between the movements in Spain and the United States. These were the Spaniards (and residents of Spain), who went to participate in the occupation of Zuccotti Park. These activists travelled to New York on their own and without any coordination. The trip was not paid for by the movement, since, unlike OWS, 15M did not receive donations and had no funds. According to the accounts of US activists collected in the interviews, these visitors contributed to the development of the movement through the transmission of a number of practical issues, which were relevant for the emergence and continuity of the movement, for example, in relation to the way to confront similar problems, such as those posed by groups that endangered coexistence among the occupiers, ${ }^{16}$ or issues such as the power supply in the camp or the design and set up of media equipment for live-streaming, among other things. ${ }^{17}$

\section{Discussion AND CONCLUSIONS}

The comparison of the diffusion from the Arab Spring to the $15 \mathrm{M}$ movement with the

16 Interview with Max Berger (26 years old), October 9, 2012, New York.

17 Interview with Nathan Schneider (age unknown, journalist), October 19, 2012, New York. diffusion from 15M to the Occupy movement in the United States reveals that what was transmitted and the type of links through which diffusion took place differed in the two processes. In the first process, the Spanish indignados received a strong sense of the efficacy of collective action carried out by the Arab protestors, especially in Egypt. The diffusion of this element took place through the publicity the protest received in the media and social networks and in the absence of interpersonal contacts. Thus, the diffusion was indirect (Tarrow, 2005). Once it was received, the agency component of collective action frames was influenced by the permissive response of the authorities and the hope for change fostered by the scale and the support the movement itself achieved.

In any case, the diffusion from Egypt to Spain was not only ideational (Givan, Roberts and Soule, 2010), in relation to the agency component of the collective action frames. It also included forms of action, although in this case, the indignados only received their general outlines. The Spanish activists replicated the general form of the protest in Egypt: occupying the main square of the city with the aim of achieving a certain permanence. Again, the diffusion took place through the media (old and new). However, beyond the general outlines of this modality of protest (Patel, 2013), the occupation of Tahrir and the dozens of occupations of Spanish squares were to a large degree different. The differences can be partly explained in relation to local contention and collective learning processes associated with the experiences of recent social protests (Romanos, 2013). The comparison of this particular process of diffusion with the one that took place between Spain and the United States suggests that these differences also have to do with the absence of interpersonal contact in the process of communicating more behavioural elements (Givan, Roberts and Soule, 2010).

The analysis of the diffusion from $15 \mathrm{M}$ to OWS reveals the transmission of innovations 
related to forms of action and organisation. The US activists received a somewhat novel form of organisational inclusiveness, one that targeted potential participants in the movement and aimed at transforming public space into a space open to empathy. Other items diffused were related to ways of organising deliberation, the space and the activity taking place in the encampment so that it would become an operational space capable of attracting potential supporters and of keeping participants active in the creation of networks and projects. These items were diffused with the help of Spanish emigrants residing in New York, who participated in both the prior organisation and subsequent development of OWS.

The comparison of these two diffusion processes helps to further our understanding of the transnational diffusion of protest in different ways. First, the comparison provides elements that help to show to what degree a certain type of channel is more likely to facilitate the diffusion of a certain item (Soule, 2004). The diffusion of the Arab Spring to $15 \mathrm{M}$ was not only ideational; however, the transmission of behavioural elements was limited. The diffusion from 15M to OWS not only take place through the action of mediators, in this case, the Spanish emigrants, but it was also supported by information in the media. But it was precisely the interpersonal contact that made it possible to transmit somewhat complex behavioural innovations. The comparison of the two processes suggests a hypothesis regarding the relationship between the character of the item to be diffused and the mechanisms of diffusion: ideational elements are diffused easily through indirect channels, whereas behavioural innovations require interpersonal contact to be properly diffused. The findings of this study indicate that the agency component of the collective action frames can be transmitted in absence of direct contact between the transmitters and the receivers of the diffusion, or when there is no one who can put them in contact; in contrast, other more com- plex items related to the organisation of social movements and the development of collective action repertoires specifically need these interpersonal links. Future studies will be needed to confirm to what degree this dynamic can also be observed in other processes of diffusion. If confirmed, this hypothesis raises questions about those studies that have emphasised the role of new media in the diffusion of social movements and protest (Tremayne, 2014; Vasi and Suh, 2013; Castells, 2012; Ayres, 1999).

Comparative analysis of the two processes also allows us suggest answers to other questions recently posed by Soule (2013), among them: 1) whether the mechanisms and processes of diffusion are the same with both ideational and behavioural innovations; ii) whether these innovations are transmitted with a greater or lesser degree of adaptation and; iii) how effective are direct and indirect models in spreading social movements. The affinity suggested above between indirect channels and ideational elements and between the presence of interpersonal contact and the diffusion of behavioural elements seems to be related to the effectiveness of different channels in the transmission of one type of element or another. Activists learn about forms of action and organisation used in other countries by reading about them or watching them on Youtube. This is what happened with the diffusion of the occupations that went from Egypt to Spain and the United States. However, the findings of this study show that the diffusion was only like this in relation to the general form of the occupation. The comparison of the two processes suggests that the specific content of the occupation - how to occupy and how to give meaning to the occupation - was not transmitted in the first process but rather in the second, precisely through the involvement of third parties. The almost instant availability of written information and videos was undoubtedly important for the transnational diffusion of the protest from Egypt to Spain, but analysis of the 
second diffusion process from Spain to the US suggests that the participation of mediators proved effective in transmitting specific knowledge and techniques, which would surely have been more difficult to grasp without the presence of activists to explain them in detail and advocate for their use.

If local experiences were important when occupations were launched in Spain (Romanos, 2013), why should that not be the case in New York? Undoubtedly it was, and hence, the importance of the Spanish emigrants, who had been living in the city for a long time and who were therefore fairly familiar with the local environment. Local contention influenced the reception of innovations, which were adopted with some adaptation. The Spanish emigrants specifically faced problems related to the translation and adaptation of certain innovations. But the difference here was the availability and involvement of transnational activists - in this case Spanish immigrants - in the diffusion process. The accounts collected in this study suggest that there was not the same level of involvement of Egyptian emigrants in the first process. At the same time, the action of the Spanish emigrants in New York occurred in a context of intense mobilisation that attracted a lot of newcomers who did not have previous experience in social movements, and as a result, were less resistant to proposals coming from the outside. Thus, the ever present process of cultural adaptation was relatively less intense than in other cases of diffusion (Wood, 2012; Givan, Roberts and Soule, 2010).

\section{Bibliography}

Álvarez, Klaudia et al. (2011). Nosotros, los indignados. Barcelona: Destino.

Ayres, J. M. (1999). "From the Streets to the Internet: The Cyber-Diffusion of Contention". Annals of the American Academy of Political and Social Science, 566: 132-143.

Castells, Manuel (2012). Redes de indignación y esperanza. Madrid: Alianza.
Chabot, Sean (2010). "Dialogue Matters: Beyond the Transmission Model of Transnational Difussion between Social Movements". In: Givan, R. K.; Roberts, K. M. and Soule, S. A. (eds). The Diffusion of Social Movements. Cambridge: Cambridge University Press.

Della Porta, Donatella (2005). "Making the Polis: Social Forums and Democracy in the Global Justice Movement". Mobilization, 10(1): 73-94.

Della Porta, Donatella and Diani, Mario (2011). Los Movimientos Sociales. Madrid: CIS-UCM.

Diani, Mario (2011). "Networks and Internet into Perspective". Swiss Political Science Review, 17(4): 469-474.

Doerr, Nicole (2008). "Deliberative Discussion, Language, and Efficiency in the WSF Process". Mobilization, 13(4): 395-410.

Flesher Fominaya, Cristina (2014). Social Movements and Globalization. New York: Palgrave.

Gamson, William (2011). "Arab Spring, Israeli Summer, and the Process of Cognitive Liberation". Swiss Political Science Review, 17(4): 463-468.

Ghonim, W. (2012). Revolution 2.0: A Memoir. London: Fourth State.

Gitlin, Todd (2012). Occupy Nation. New York: Harper Collins.

Givan, Rebecca Kolins; Roberts, Kenneth M. and Soule, Sarah A. (2010). The Diffusion of Social Movements. Cambridge: Cambridge University Press.

Goldstone, Jack (2011). "Cross-class Coalitions and the Making of the Arab Revolts of 2011". Swiss Political Science Review, 17(4): 457-462.

Gordon, Uri (2012). “Israel's 'Tent Protests': The Chilling Effect of Nationalism". Social Movement Studies, 11(3-4): 349-355.

Graeber, David (2011). "On Playing by the Rules: The Strange Success of \#OccupyWallStreet". Naked Capitalism, Available at: http://www.nakedcapitalism.com/2011/10/david-graeber-on-playingby-the-rules- --the-strange-success-of-occupywall-street.html, consulted on November 30, 2012.

Hammond, John (2013). "The Significance of Space in Occupy Wall Street". Interface, 5(2): 499-524.

Kerton, Sarah (2012). "Tahrir, Here? The Influence of the Arab Uprisings on the Emergence of Occupy". Social Movement Studies, 11(3-4): 302-308. 
Kroll, Andy (2011). "How Occupy Wall Street Really Got Started". In: van Gelder, S. (ed.). This Changes Everything. San Francisco, California: BerrettKoehler Publishers.

Kurzman, Charles (2012). "The Arab Spring Uncoiled". Mobilization, 17(4): 377-390.

Lawrence, Jeffrey (2013). "The International Roots of the $99 \%$ and the 'Politics of anyone'”. IC - Revista Científica de Información y Comunicación, 10: $53-72$.

Muñoz, Alba (2011). "Del síndrome Wikileaks a la democracia 2.0. Las redes sociales y el 15-M". In: Antentas, J. M. et al. (eds). Las Voces del 15$M$. Barcelona: Los libros del lince.

Naidu, Suresh (2011). "My Trajectory with Occupy". In: Schiffrin, A. and Kircher-Allen, E. (eds). From Cairo to Wall Street. New York: The New Press.

Patel, David S. (2013). "Roundabouts and Revolutions: Public Squares, Coordination, and the Diffusion of the Arab Uprisings". Unpublished paper.

Rogers, E. M. (1995). Diffusion of Innovations. (4th edition) New York: Free Press.

Romanos, Eduardo (2011a). "El 15M y la democracia de los movimientos sociales". Books and Ideas, October 18.

Romanos, Eduardo (2011b). "Emociones, identidad y represión: el activismo anarquista durante el franquismo". Revista Española de Investigaciones Sociológicas, 134: 87-106.

Romanos, Eduardo (2013). "Collective Learning Processes within Social Movements: Some Insights into the Spanish 15M/Indignados Movement". In: Flesher Fominaya, C. and Cox, L. (eds). Understanding European Movements. London: Routledge.

Sitrin, Marina and Azzellini, Dario (2012). Occupying Language. New York: Occupied Media Pamphlet Series.

Snow, David. A. and Benford, Robert. D. (1992). "Master Frames and Cycles of Protest". In: Morris, A. and Mueller, C. (eds). Frontiers of Social Movement Theory. New Haven, Connecticut: Yale University Press.
Soule, Sarah A. (2004). "Diffusion Processes within and across Movements". In: Snow, D. A.; Soule, S. A. and Kriesi, H. (eds). The Blackwell Companion to Social Movements. Malden, Massachusetts: Blackwell.

Soule, Sarah A. (2013). "Diffusion and Scale Shift". In: Snow, D. A. et al. (eds). The Wiley-Blackwell Encyclopedia of Social and Political Movements. Malden, Massachusetts: Blackwell.

Tarrow, Sidney (1989). Democracy and Disorder: Protest and Politics in Italy, 1965-1974. New York: Oxford University Press.

Tarrow, Sidney (2005). The New Transnational Activism. Cambridge: Cambridge University Press.

Tejerina, Benjamín et al. (2013). "From Indignation to Occupation: A New Wave of Global Mobilization". Current Sociology, 61(4): 377-392.

Tilly, Charles and Tarrow, Sidney (2007). Contentious Politics. Boulder, Colorado: Paradigm.

Toret, Javier (2012). "Una mirada tecnopolítica sobre los primeros días del \#15M". In: Alcazan et al. (eds). Tecnopolítica, internet y r-evoluciones. Barcelona: Icaria.

Tremayne, M. (2014). "Anatomy of Protest in the Digital Era: A Network Analysis of Twitter and Occupy Wall Street". Social Movement Studies, 13(1): 110-126.

Vasi, Ion Bogdan and Suh, Chan S. (2012). "Protest in the Internet Age: Public Attention, Social Media, and the Spread of the 'Occupy' Movement in the United States". Paper presented at the American Sociological Association Annual Conference, Denver, Colorado.

Voulgarelis, Antonis (2012). "Nights in Syntagma Square". In: Schiffrin, A. and Kircher-Allen, E. (eds). From Cairo to Wall Street. New York: The New Press.

Walsh-Russo, C. (2014). "Diffusion of Protest". Sociology Compass, 8(1): 31-42.

Wood, Lesley J. (2012). Direct Action, Deliberation, and Diffusion: Collective Action after the WTO Protests in Seattle. Cambridge: Cambridge University Press.

RECEPTION: October 29, 2014

REVIEW: March 11, 2015

ACCEPTANCE: May 25, 2015

Reis. Rev.Esp.Investig.Sociol. ISSN-L: 0210-5233. № 154, April - June 2016, pp. 103-118 
